\title{
Article
}

\section{Global Bounds for the Generalized Jensen Functional with Applications}

\author{
Slavko Simić ${ }^{1, * \mathbb{D}}$ and Bandar Bin-Mohsin ${ }^{2}$ \\ 1 Mathematical Institute SANU, 11000 Belgrade, Serbia \\ 2 Department of Mathematics, College of Science, King Saud University, Riyadh 11451, Saudi Arabia; \\ balmohsen@ksu.edu.sa \\ * Correspondence: ssimic@turing.mi.sanu.ac.rs
}

Citation: Simić, S.; Bin-Mohsin, B. Global Bounds for the Generalized Jensen Functional with Applications. Symmetry 2021, 13, 2105. https:// doi.org/10.3390/sym13112105

Academic Editor: Nicusor Minculete

Received: 17 October 2021 Accepted: 30 October 2021

Published: 6 November 2021

Publisher's Note: MDPI stays neutral with regard to jurisdictional clai$\mathrm{ms}$ in published maps and institutional affiliations.

Copyright: ( 2021 by the authors. Licensee MDPI, Basel, Switzerland. This article is an open access article distributed under the terms and conditions of the Creative Commons Attribution (CC BY) license (https:// creativecommons.org/licenses/by/ $4.0 /)$.
Abstract: In this article we give sharp global bounds for the generalized Jensen functional $J_{n}(g, h ; \mathbf{p}, x)$. In particular, exact bounds are determined for the generalized power mean in terms from the class of Stolarsky means. As a consequence, we obtain the best possible global converses of quotients and differences of the generalized arithmetic, geometric and harmonic means.

Keywords: Jensen functional; A-G-H inequalities; global bounds; power means; convex functions

MSC: 26D07(26D15)

\section{Introduction}

Recall that the Jensen functional $J_{n}(h ; \mathbf{p}, \mathbf{x})$ is defined on an interval $I \subseteq \mathbb{R}$ by

$$
J_{n}(h ; \mathbf{p}, x):=\sum_{1}^{n} p_{i} h\left(x_{i}\right)-h\left(\sum_{1}^{n} p_{i} x_{i}\right),
$$

where $h: I \rightarrow \mathbb{R}, \mathbf{x}=\left(x_{1}, x_{2}, \cdots, x_{n}\right) \in I^{n}$ and $\mathbf{p}=\left\{p_{i}\right\}_{1}^{n}$ is a positive weight sequence.

If $h$ is a convex function on $I$ then the inequality

$$
0 \leq J_{n}(h ; \mathbf{p}, x)
$$

holds for each $\mathbf{x} \in I^{n}$ and any positive weight sequence $\mathbf{p}$.

If $h$ is a concave function on $I$ then the above inequality is reversed. Those inequalities play a fundamental role in many parts of mathematical analysis and applications. For example, the well-known $\mathcal{A}-\mathcal{G}-\mathcal{H}$ inequality, Holder's inequality, Ky Fan inequality, etc., are proven by the help of Jensen's inequality (cf. [1-6]).

Our aim in this paper is to find the simplest constant $C$ such that

$$
0 \leq J_{n}(h ; \mathbf{p}, x) \leq C
$$

for any choice of $\mathbf{p}, x$ and thus make this inequality symmetrical.

This will be done by assuming that $\mathbf{x} \in[a, b]^{n} \subset I^{n}$, and we shall find some global bounds for the generalized Jensen functional

$$
J_{n}(g, h ; \mathbf{p}, x):=g\left(\sum_{1}^{n} p_{i} h\left(x_{i}\right)\right)-g\left(h\left(\sum_{1}^{n} p_{i} x_{i}\right)\right),
$$

that is, the bounds not depending on $\mathbf{p}$ or $\mathbf{x}$ but only on $a, b$ and functions $g$ and $h$. In this sense, a typical result is given by the part of Theorem 1 (below). 
For $\mathbf{x} \in[a, b]^{n} \subset I^{n}$, let $h: I \rightarrow J$ be convex and $g: J \rightarrow \mathbb{R}$ be an increasing function. Then

$$
0 \leq J_{n}(g, h ; \mathbf{p}, x) \leq \max _{p}[g(p h(a)+(1-p) h(b))-g(h(p a+(1-p) b))] .
$$

Our global bounds will be entirely presented in terms of elementary means.

Recall that the mean is a map $M: \mathbb{R}_{+} \times \mathbb{R}_{+} \rightarrow \mathbb{R}_{+}$, with a property

$$
\min (x, y) \leq M(x, y) \leq \max (x, y)
$$

for each $x, y \in \mathbb{R}_{+}$.

In the sequel we shall use the class of so-called Stolarsky (or extended) two-parametric mean values, defined for positive values of $x, y, x \neq y$ by the following

$$
E_{r, s}(x, y)= \begin{cases}\left(\frac{r\left(x^{s}-y^{s}\right)}{s\left(x^{r}-y^{r}\right)}\right)^{1 /(s-r)}, & r s(r-s) \neq 0 \\ \exp \left(\frac{-1}{s}+\frac{x^{s} \log x-y^{s} \log y}{x^{s}-y^{s}}\right), & r=s \neq 0 \\ \left(\frac{x^{s}-y^{s}}{s(\log x-\log y)}\right)^{1 / s}, & s \neq 0, r=0 \\ \sqrt{x y}, & r=s=0 \\ x, & y=x>0 .\end{cases}
$$

In this form it was introduced by Keneth Stolarsky in [7].

Most of the classical two variable means are special cases of the class E. For example,

$$
A(x, y)=E_{1,2}(x, y)=\frac{x+y}{2}
$$

is the arithmetic mean;

$$
G(x, y)=E_{0,0}(x, y)=E_{-r, r}(x, y)=\sqrt{x} y
$$

is the geometric mean;

$$
L(x, y)=E_{0,1}(x, y)=\frac{x-y}{\log x-\log y}
$$

is the logarithmic mean;

$$
I(x, y)=E_{1,1}(x, y)=\left(x^{x} / y^{y}\right)^{\frac{1}{x-y}} / e
$$

is the identric mean, etc.

More generally, the $r$-th power mean

$$
A_{r}(x, y)=\left(\frac{x^{r}+y^{r}}{2}\right)^{1 / r}
$$

is equal to $E_{r, 2 r}(x, y)$.

Using the class of Stolarsky means enables our results to be presented in a condensed and applicable way. For example, we give some results regarding $\mathcal{A}-\mathcal{G}-\mathcal{H}$ inequalities, where

$$
\begin{gathered}
\mathcal{A}(\mathbf{p}, x):=\sum_{1}^{n} p_{i} x_{i} \\
\mathcal{G}(\mathbf{p}, x):=\prod_{1}^{n} x_{i}^{p_{i}} \\
\mathcal{H}(\mathbf{p}, x):=\left(\sum_{1}^{n} p_{i} / x_{i}\right)^{-1}
\end{gathered}
$$


are the generalized arithmetic, geometric and harmonic means, respectively.

Let $\mathbf{x} \in[a, b]^{n}, 0<a<b$. Then

$$
\begin{gathered}
0 \leq \mathcal{A}(\mathbf{p}, x)-\mathcal{H}(\mathbf{p}, x) \leq 2(A(a, b)-G(a, b)) \\
0 \leq \mathcal{A}(\mathbf{p}, x)-\mathcal{G}(\mathbf{p}, x) \leq A(a, b)-L(a, b)+L(a, b) \log \frac{L(a, b)}{G(a, b)} \\
1 \leq \frac{\mathcal{A}(\mathbf{p}, x)}{\mathcal{H}(\mathbf{p}, x)} \leq\left(\frac{A(a, b)}{G(a, b)}\right)^{2} ; \\
1 \leq \frac{\mathcal{G}(\mathbf{p}, x)}{\mathcal{H}(\mathbf{p}, x)} \leq \frac{I(a, b) L(a, b)}{G^{2}(a, b)} \\
1 \leq \frac{\mathcal{A}(\mathbf{p}, x)}{\mathcal{G}(\mathbf{p}, x)} \leq \frac{I(a, b) L(a, b)}{G^{2}(a, b)}
\end{gathered}
$$

where $A, G, H, L, I$ stands for the arithmetic, geometric, harmonic, logarithmic and identric means of positive numbers $a$ and $b$, respectively.

All bounds above are the best possible.

\section{Results and Proofs}

Our results concerning global bounds for the generalized Jensen functional are given in the following two assertions.

Theorem 1. 1. For continuous functions $g$, $h$ let $h: I \rightarrow J$ be convex and $g: J \rightarrow \mathbb{R}$ be an increasing function or $h: I \rightarrow J$ be concave and $g: J \rightarrow \mathbb{R}$ be a decreasing function. Then

$$
0 \leq J_{n}(g, h ; p, x) \leq \max _{p}[g(p h(a)+(1-p) h(b))-g(h(p a+(1-p) b))] .
$$

2. If $h: I \rightarrow J$ is convex and $g: J \rightarrow \mathbb{R}$ is a decreasing function or $h: I \rightarrow J$ is concave and $g: J \rightarrow \mathbb{R}$ is an increasing function. Then

$$
0 \leq-J_{n}(g, h ; p, x) \leq \max _{p}[g(h(p a+(1-p) b))-g(p h(a)+(1-p) h(b))] .
$$

Proof. We shall prove only the part 1 . The proof of part 2 of this theorem is analogous.

Therefore, if $h$ is a convex function on $J$ we have $\sum_{1}^{n} p_{i} h\left(x_{i}\right) \geq h\left(\sum_{1}^{n} p_{i} x_{i}\right)$. Since $g$ is an increasing function, it follows that

$$
J_{n}(g, h ; \mathbf{p}, x)=g\left(\sum_{1}^{n} p_{i} h\left(x_{i}\right)\right)-g\left(h\left(\sum_{1}^{n} p_{i} x_{i}\right)\right) \geq 0 .
$$

Similarly, if $h$ is a concave function on $J$ we have $\sum_{1}^{n} p_{i} h\left(x_{i}\right) \leq h\left(\sum_{1}^{n} p_{i} x_{i}\right)$. Since $g$ is a decreasing function, it follows again that

$$
J_{n}(g, h ; \mathbf{p}, x) \geq 0 .
$$

On the other hand, since $a \leq x_{i} \leq b$, there exist non-negative numbers $\lambda_{i}, \mu_{i} ; \lambda_{i}+\mu_{i}=$ 1 , such that $x_{i}=\lambda_{i} a+\mu_{i} b, i=1,2, \ldots, n$.

Hence,

$$
\begin{gathered}
J_{n}(g, h ; \mathbf{p}, x)=g\left(\sum_{1}^{n} p_{i} h\left(x_{i}\right)\right)-g\left(h\left(\sum_{1}^{n} p_{i} x_{i}\right)\right)=g\left(\sum_{1}^{n} p_{i} h\left(\lambda_{i} a+\mu_{i} b\right)\right)-g\left(h\left(\sum_{1}^{n} p_{i}\left(\lambda_{i} a+\mu_{i} b\right)\right)\right) \\
\left.\leq g\left(\sum_{1}^{n} p_{i}\left(\lambda_{i} h(a)+\mu_{i} h(b)\right)\right)-g\left(h\left(a \sum_{1}^{n} p_{i} \lambda_{i}+b \sum_{1}^{n} p_{i} \mu_{i}\right)\right)\right)
\end{gathered}
$$




$$
=g(p h(a)+(1-p) h(b))-g(h(p a+(1-p) b)):=F(p ; a, b) \leq \max _{p} F(p ; a, b),
$$

where we denoted $\sum_{1}^{n} p_{i} \lambda_{i}:=p \in[0,1]$.

The second case with concave $h$ and decreasing $g$ leads to the same result.

Note that the function $F(p ; a, b)$ is continuous in $p$ and non-negative with $F(0 ; a, b)=$ $F(1 ; a, b)=0$. Therefore, $\max _{p} F(p ; a, b)$ exists. Another and sometimes difficult problem is to evaluate its exact value (see Open Problem below).

For this cause, we give an estimation of $J_{n}(g, h ; \mathbf{p}, x)$ with a unique maximum, which could be easily calculated. This method can be applied to the second part of Theorem 1 , as well.

Theorem 2. 1. Under the conditions of the first part of Theorem 1, assume firstly that $g$ is a convex function on $\mathrm{J}$. Then

$$
0 \leq J_{n}(g, h ; p, x) \leq \max _{p}[p f(a)+(1-p) f(b)-f(p a+(1-p) b)],
$$

where $f:=g \circ h$.

2. Assuming that $f=g \circ h$ is a concave function, we have

$$
0 \leq J_{n}(g, h ; p, x) \leq \max _{p}[g(p h(a)+(1-p) h(b))-(p f(a)+(1-p) f(b))] .
$$

Now, both maximums can be easily determined by the standard technique.

Proof. By the first part of Theorem 1 , we found that there exists $p \in[0,1]$ such that

$$
J_{n}(g, h ; \mathbf{p}, x) \leq g(p h(a)+(1-p) h(b))-g(h(p a+(1-p) b)) .
$$

If additionally $g$ is convex on $J$, then

$$
g(p h(a)+(1-p) h(b)) \leq p(g \circ h)(a)+(1-p)(g \circ h)(b) .
$$

Hence,

$$
\begin{gathered}
\left.J_{n}(g, h ; \mathbf{p}, x) \leq p(g \circ h)(a)+(1-p)(g \circ h)(b)-(g \circ h)(p a+(1-p) b)\right) \\
=p f(a)+(1-p) f(b)-f(p a+(1-p) b)] \leq \max _{p}[p f(a)+(1-p) f(b)-f(p a+(1-p) b)] .
\end{gathered}
$$

Consequently, if $g \circ h$ is a concave function on $J$, we have

$$
g(h(p a+(1-p) b)=(g \circ h)(p a+(1-p) b) \geq p(g \circ h)(a)+(1-p)(g \circ h)(b),
$$

and

$$
J_{n}(g, h ; \mathbf{p}, x) \leq \max _{p}[g(p h(a)+(1-p) h(b))-(p f(a)+(1-p) f(b))] .
$$

\section{Applications}

The results above are the source of a number of interesting inequalities. For instance, taking $g(x)=\log x$ in Theorem 1 , we are enabled to determine converses of the quotient

$$
\frac{\sum p_{i} h\left(x_{i}\right)}{h\left(\sum p_{i} x_{i}\right)}
$$

Or, taking $g(x)=h^{-1}(x)$, we can estimate the difference

$$
\mathcal{A}_{h}(\mathbf{p}, x)-\mathcal{A}(\mathbf{p}, x)
$$


where

$$
\mathcal{A}_{h}(\mathbf{p}, x):=h^{-1}\left(\sum p_{i} h\left(x_{i}\right)\right)
$$

is the quasi-arithmetic mean and

$$
\mathcal{A}_{x}(\mathbf{p}, x)=\mathcal{A}(\mathbf{p}, x)=\sum p_{i} x_{i}
$$

is the generalized arithmetic mean.

We shall specialize this argument for the class of generalized power means $\mathcal{B}_{S}(\mathbf{p}, x)$ of order $s \in \mathbb{R}$, where

$$
\mathcal{B}_{s}(\mathbf{p}, x):=\left(\sum p_{i} x_{i}^{S}\right)^{1 / s} .
$$

Some important particular cases are

$$
\begin{gathered}
\mathcal{B}_{-1}(\mathbf{p}, x)=\left(\sum_{1}^{n} p_{i} / x_{i}\right)^{-1}:=\mathcal{H}(\mathbf{p}, x) ; \\
\mathcal{B}_{0}(\mathbf{p}, x)=\lim _{s \rightarrow 0} \mathcal{B}_{\mathcal{S}}(\mathbf{p}, x)=\prod_{1}^{n} x_{i}^{p_{i}}:=\mathcal{G}(\mathbf{p}, x) ; \\
\mathcal{B}_{1}(\mathbf{p}, x)=\sum_{1}^{n} p_{i} x_{i}:=\mathcal{A}(\mathbf{p}, x),
\end{gathered}
$$

that is, the generalized harmonic, geometric and arithmetic means, respectively.

It is well-known that $\mathcal{B}_{s}(\mathbf{p}, x)$ is monotone increasing in $s \in \mathbb{R}$ (cf. [4]).

Therefore,

$$
\mathcal{H}(\mathbf{p}, x) \leq \mathcal{G}(\mathbf{p}, x) \leq \mathcal{A}(\mathbf{p}, x),
$$

represents the famous $\mathcal{A}-\mathcal{G}-\mathcal{H}$ inequality.

As an application of Theorem 1 , we shall estimate the difference $\mathcal{B}_{s}(\mathbf{p}, x)-\mathcal{A}(\mathbf{p}, x)$.

Theorem 3. Let $\mathbf{x} \in[a, b]^{n} \subset I^{n}, 0<a<b$.

Then

$$
\begin{aligned}
0 & \leq \mathcal{B}_{s}(\boldsymbol{p}, x)-\mathcal{A}(\boldsymbol{p}, x) \leq \frac{s-1}{s}\left(E_{s, 1}(a, b)-E_{s, s-1}^{-1}(1 / a, 1 / b)\right), s>1 ; \\
0 & \leq \mathcal{A}(\boldsymbol{p}, x)-\mathcal{B}_{s}(\boldsymbol{p}, x) \leq \frac{1-s}{s}\left(E_{1, s}(a, b)-E_{1-s,-s}(a, b)\right), 0<s<1 ; \\
& 0 \leq \mathcal{A}(\boldsymbol{p}, x)-\mathcal{B}_{s}(\boldsymbol{p}, x) \leq \frac{s-1}{s}\left(E_{1-s,-s}(a, b)-E_{1, s}(a, b)\right), s<0 .
\end{aligned}
$$

Proof. Let $h(x)=x^{s}, g(x)=x^{1 / s}, s \in \mathbb{R} /\{0\}$.

If $s>1$, then $h$ is a convex function and $g$ is monotone increasing on $(0, \infty)$. Hence, by the first part of Theorem 1, we obtain

$$
0 \leq \mathcal{B}_{s}(\mathbf{p}, x)-\mathcal{A}(\mathbf{p}, x) \leq \max _{p}\left(\left(p a^{s}+(1-p) b^{s}\right)^{1 / s}\right)-(p a+(1-p) b):=M_{\mathcal{S}}\left(p_{0} ; a, b\right) .
$$

This maximum is easy to calculate and we obtain

$$
p_{0} a^{s}+\left(1-p_{0}\right) b^{s}=\left(\frac{b^{s}-a^{s}}{s(b-a)}\right)^{s /(s-1)}=E_{s, 1}^{s}(a, b) .
$$

Therefore,

$$
p_{0}=\frac{b^{s}-E_{s, 1}^{s}(a, b)}{b^{s}-a^{s}} ; 1-p_{0}=\frac{E_{s, 1}^{s}(a, b)-a^{s}}{b^{s}-a^{s}},
$$

and

$$
p_{0} a+\left(1-p_{0}\right) b=\frac{a b^{s}-b a^{\mathcal{S}}}{b^{\mathcal{S}}-a^{s}}+\frac{b-a}{b^{\mathcal{S}}-a^{s}} E_{s, 1}^{s}(a, b) .
$$


Since,

$$
E_{s, 1}^{s}(a, b)=\frac{b^{s}-a^{s}}{s(b-a)} E_{s, 1}(a, b),
$$

we obtain

$$
\begin{gathered}
M_{s}\left(p_{0} ; a, b\right)=\left(p_{0} a^{s}+\left(1-p_{0}\right) b^{s}\right)^{1 / s}-\left(p_{0} a+\left(1-p_{0}\right) b\right) \\
=E_{s, 1}(a, b)-\left(\frac{(1 / a)^{s-1}-(1 / b)^{s-1}}{(1 / a)^{s}-(1 / b)^{s}}+\frac{1}{s} E_{s, 1}(a, b)\right) \\
=\frac{s-1}{s}\left(E_{s, 1}(a, b)-E_{s, s-1}^{-1}(1 / a, 1 / b)\right) .
\end{gathered}
$$

In cases $0<s<1$ and $s<0$ one should apply the second part of Theorem 1, since then $h$ is concave and $g$ is increasing in the first case and $h$ is convex and $g$ is decreasing in the second case. Proceeding as above, the result follows.

As a consequence, we obtain some converses of the $\mathcal{A}(\mathbf{p}, x)-\mathcal{G}(\mathbf{p}, x)-\mathcal{H}(\mathbf{p}, x)$ inequality.

Corollary 1. Let $\mathbf{x} \in[a, b]^{n} \subset I^{n}, b>a>0$.

Then

$$
0 \leq \mathcal{A}(p, x)-\mathcal{H}(p, x) \leq 2(A(a, b)-G(a, b)) .
$$

Proof. Putting $s=-1$, we obtain

$$
\begin{gathered}
0 \leq \mathcal{A}(\mathbf{p}, x)-\mathcal{B}_{-1}(\mathbf{p}, x)=\mathcal{A}(\mathbf{p}, x)-\mathcal{H}(\mathbf{p}, x) \\
\leq 2\left(E_{2,1}(a, b)-E_{1,-1}(a, b)\right)=2(A(a, b)-G(a, b)) .
\end{gathered}
$$

Corollary 2. Let $\mathbf{x} \in[a, b]^{n} \subset I^{n}, b>a>0$.

Then

$$
0 \leq \mathcal{A}(\boldsymbol{p}, x)-\mathcal{G}(\boldsymbol{p}, x) \leq L(a, b) \log \frac{L(a, b) I(a, b)}{G^{2}(a, b)} .
$$

Proof. Letting $s \rightarrow 0$, we have

$$
\begin{gathered}
\mathcal{A}(\mathbf{p}, x)-\mathcal{G}(\mathbf{p}, x)=\lim _{s \rightarrow 0}\left(\mathcal{A}(\mathbf{p}, x)-\mathcal{B}_{s}(\mathbf{p}, x)\right) \\
\leq \lim _{s \rightarrow 0}\left(\frac{1-s}{s}\left(E_{1, s}(a, b)-E_{1-s,-s}(a, b)\right)\right) .
\end{gathered}
$$

After somewhat laborous calculation using Taylor series, the result follows.

Remark 1. Estimating the Jensen functional

$$
J_{n}\left(e^{x} ; \mathbf{p}, \mathbf{x}\right)=\sum_{1}^{n} p_{i} e^{x_{i}}-e^{\sum_{1}^{n} p_{i} x_{i}}
$$

for $\mathbf{x} \in[a, b]^{n} \subset \mathbb{R}^{n}$, and then changing variables $x_{i} \rightarrow \log x_{i} ; a \rightarrow \log a, b \rightarrow \log b$, we obtain the same result.

Open problem Find the exact upper global bound for

$$
\mathcal{G}(p, x)-\mathcal{H}(p, x) \text {. }
$$

The next proposition gives global bounds for the quotient of two power means. 
Theorem 4. For $s>t$ and $\mathbf{x} \in[a, b]^{n} \subset \mathbb{R}_{+}^{n}$, we have

$$
1 \leq \frac{\mathcal{B}_{s}(\boldsymbol{p}, x)}{\mathcal{B}_{t}(\boldsymbol{p}, x)} \leq \frac{E_{s, s-t}(a, b)}{E_{t, t-s}(a, b)}
$$

Both bounds are the best possible.

Proof. Applying the method from the proof of Theorem 1, we obtain

$$
x_{i}^{t}=\lambda_{i} a^{t}+\mu_{i} b^{t}, \lambda_{i}+\mu_{i}=1, i=1,2, \ldots, n .
$$

In the cases $s>t>0$ or $s>0, t<0$, we have that the function $x^{s / t}$ is convex. Hence,

$$
x_{i}^{s}=\left(\lambda_{i} a^{t}+\mu_{i} b^{t}\right)^{s / t} \leq \lambda_{i}\left(a^{t}\right)^{s / t}+\mu_{i}\left(b^{t}\right)^{s / t}=\lambda_{i} a^{s}+\mu_{i} b^{t},
$$

and

$$
\frac{\mathcal{B}_{s}(\mathbf{p}, x)}{\mathcal{B}_{t}(\mathbf{p}, x)}=\frac{\left(\sum_{1}^{n} p_{i} x_{i}^{s}\right)^{1 / s}}{\left(\sum_{1}^{n} p_{i} x_{i}^{t}\right)^{1 / t}} \leq \frac{\left(a^{s} \sum_{1}^{n} p_{i} \lambda_{i}+b^{s} \sum_{1}^{n} p_{i} \mu_{i}\right)^{1 / s}}{\left(a^{t} \sum_{1}^{n} p_{i} \lambda_{i}+b^{t} \sum_{1}^{n} p_{i} \mu_{i}\right)^{1 / t}}=\frac{\left(p a^{s}+q b^{s}\right)^{1 / s}}{\left(p a^{t}+q b^{t}\right)^{1 / t}}
$$

where we put

$$
\sum_{1}^{n} p_{i} \lambda_{i}:=p, \sum_{1}^{n} p_{i} \mu_{i}:=q ; p+q=1
$$

Therefore, it follows that

$$
\frac{\mathcal{B}_{s}(\mathbf{p}, x)}{\mathcal{B}_{t}(\mathbf{p}, x)} \leq \max _{p} \frac{\left(p a^{s}+q b^{s}\right)^{1 / s}}{\left(p a^{t}+q b^{t}\right)^{1 / t}}=\frac{\left(p_{0} a^{s}+q_{0} b^{s}\right)^{1 / s}}{\left(p_{0} a^{t}+q_{0} b^{t}\right)^{1 / t}}
$$

By standard means we obtain that this maximum satisfies the equation

$$
\frac{s\left(p_{0} a^{s}+q_{0} b^{s}\right)}{a^{s}-b^{s}}=\frac{t\left(p_{0} a^{t}+q_{0} b^{t}\right)}{a^{t}-b^{t}}
$$

that is,

$$
p_{0}=\frac{1}{s-t}\left(\frac{s b^{s}}{b^{s}-a^{s}}-\frac{t b^{t}}{b^{t}-a^{t}}\right) ; q_{0}=\frac{1}{s-t}\left(\frac{t a^{t}}{b^{t}-a^{t}}-\frac{s a^{s}}{b^{s}-a^{s}}\right) .
$$

Consequently,

$$
p_{0} a^{t}+q_{0} b^{t}=\frac{s}{s-t} \frac{a^{t} b^{s}-a^{s} b^{t}}{b^{s}-a^{s}}=\frac{s}{s-t} \frac{(a b)^{t}\left(b^{s-t}-a^{s-t}\right)}{b^{s}-a^{s}},
$$

and

$$
p_{0} a^{s}+q_{0} b^{s}=\frac{t}{s-t} \frac{a^{t} b^{s}-a^{s} b^{t}}{b^{t}-a^{t}}=\frac{t}{t-s} \frac{(a b)^{s}\left(b^{t-s}-a^{t-s}\right)}{b^{t}-a^{t}} .
$$

Hence,

$$
\begin{aligned}
& \left(p_{0} a^{t}+q_{0} b^{t}\right)^{1 / t}=G^{2}(a, b) / E_{s, s-t}(a, b) ; \\
& \left(p_{0} a^{s}+q_{0} b^{s}\right)^{1 / s}=G^{2}(a, b) / E_{t, t-s}(a, b),
\end{aligned}
$$

and we finally obtain

$$
\max _{p} \frac{\left(p a^{s}+q b^{s}\right)^{1 / s}}{\left(p a^{t}+q b^{t}\right)^{1 / t}}=\frac{\left(p_{0} a^{s}+q_{0} b^{s}\right)^{1 / s}}{\left(p_{0} a^{t}+q_{0} b^{t}\right)^{1 / t}}=\frac{E_{s, s-t}(a, b)}{E_{t, t-s}(a, b)} .
$$


In the third case, for $s>t>0$, we have

$$
\begin{aligned}
1 \leq \frac{\mathcal{B}_{-t}(\mathbf{p}, x)}{\mathcal{B}_{-s}(\mathbf{p}, x)}= & \frac{\mathcal{B}_{s}(\mathbf{p}, 1 / x)}{\mathcal{B}_{t}(\mathbf{p}, 1 / x)} \leq \frac{E_{s, s-t}(1 / a, 1 / b)}{E_{t, t-s}(1 / a, 1 / b)} \\
& =\frac{E_{S, s-t}(a, b)}{E_{t, t-s}(a, b)}
\end{aligned}
$$

since

$$
E_{u, v}(1 / a, 1 / b)=E_{u, v}(a, b) / G^{2}(a, b) .
$$

It is obvious that 1 is the best possible lower global bound. To prove that $M_{s, t}(a, b):=$ $E_{s, s-t}(a, b) / E_{t, t-s}(a, b)$ is also the best possible global bound, denote by $N_{s, t}(a, b)$ an arbitrary upper bound. Then the relation

$$
\frac{\mathcal{B}_{s}(\mathbf{p}, x)}{\mathcal{B}_{t}(\mathbf{p}, x)} \leq N_{s, t}(a, b),
$$

holds for any $\mathbf{p}$ and $\mathbf{x}$.

Putting $x_{1}=x_{2}=\ldots=x_{n-1}=a, x_{n}=b, p_{n}=q_{0}$, we obtain

$$
M_{s, t}(a, b)=\frac{\left(p_{0} a^{s}+q_{0} b^{s}\right)^{1 / s}}{\left(p_{0} a^{t}+q_{0} b^{t}\right)^{1 / t}}=\frac{\mathcal{B}_{s}(\mathbf{p}, x)}{\mathcal{B}_{t}(\mathbf{p}, x)} \leq N_{s, t}(a, b),
$$

and the proof is complete.

Some important consequences of this theorem are given in the following

Corollary 3. For $s>1$, we have

$$
\mathcal{A}(p, x) \leq \mathcal{B}_{\mathcal{S}}(p, x) \leq \frac{E_{s, s-1}(a, b)}{E_{1,1-s}(a, b)} \mathcal{A}(p, x) .
$$

Corollary 4. For $s>0$, we have

$$
\mathcal{G}(p, x) \leq \mathcal{B}_{s}(p, x) \leq \frac{E_{s, s}(a, b) E_{s, 0}(a, b)}{G^{2}(a, b)} \mathcal{G}(p, x) .
$$

Corollary 5. For $s>-1$, we have

$$
\mathcal{H}(\boldsymbol{p}, x) \leq \mathcal{B}_{s}(\boldsymbol{p}, x) \leq \frac{E_{s+1, s}(a, b) E_{s+1,1}(a, b)}{G^{2}(a, b)} \mathcal{H}(\boldsymbol{p}, x) .
$$

In the last two corollaries we used the identity

$$
E_{-u,-v}(a, b) E_{u, v}(a, b)=G^{2}(a, b) .
$$

Finally, putting $s=1$ in Corollary 4 and $s=0, s=1$ in Corollary 5, since $E_{2,1}(a, b)=$ $A(a, b), E_{1,0}(a, b)=L(a, b), E_{1,1}(a, b)=I(a, b)$, we obtain global converses of the $\mathcal{A}-\mathcal{G}-$ $\mathcal{H}$ inequality.

\section{Corollary 6.}

$$
\begin{gathered}
\mathcal{G}(\boldsymbol{p}, x) \leq \mathcal{A}(\boldsymbol{p}, x) \leq \frac{L(a, b) I(a, b)}{G^{2}(a, b)} \mathcal{G}(\boldsymbol{p}, x) \\
\mathcal{H}(\boldsymbol{p}, x) \leq \mathcal{A}(\boldsymbol{p}, x) \leq\left(\frac{A(a, b)}{G(a, b)}\right)^{2} \mathcal{H}(\boldsymbol{p}, x) \\
\mathcal{H}(\boldsymbol{p}, x) \leq \mathcal{G}(\boldsymbol{p}, x) \leq \frac{L(a, b) I(a, b)}{G^{2}(a, b)} \mathcal{H}(\boldsymbol{p}, x) .
\end{gathered}
$$


Therefore, a sort of tight symmetry is established for these inequalities.

\section{Conclusions}

We give a method for two-sided estimations of the generalized Jensen functional $J_{n}(g, h ; \mathbf{p}, \mathbf{x})$, with applications to the general means. In particular, sharp converses of the famous

$\mathcal{A}-\mathcal{G}-\mathcal{H}$ inequality are obtained. Further investigations can be undertaken on more general settings, i.e., $J_{n}(f, g, h ; \mathbf{p}, \mathbf{x}):=f\left(\sum_{1}^{n} p_{i} h\left(x_{i}\right)\right)-g\left(h\left(\sum_{1}^{n} p_{i} x_{i}\right)\right)$ or even $F\left(\sum_{1}^{n} p_{i} h\left(x_{i}\right)\right.$, $\left.h\left(\sum_{1}^{n} p_{i} x_{i}\right)\right)$, with properly chosen functions $f, g, h$ and $F(x, y)$.

Author Contributions: Theoretical part, S.S.; numerical part with examples, B.B.-M. All authors have read and agreed to the published version of the manuscript.

Funding: Bandar Bin-Mohsin is supported by Researchers Supporting Project number (RSP-2021/158), King Saud University, Riyadh, Saudi Arabia.

Institutional Review Board Statement: Not applicable.

Informed Consent Statement: Not applicable.

Data Availability Statement: Not applicable.

Acknowledgments: The authors are grateful to the referees for their valuable comments.

Conflicts of Interest: The authors declare no conflict of interests.

\section{References}

1. Hardy, G.H.; Littlewood, J.E.; Polya, G. Inequalities; Cambridge University Press: Cambridge, UK, 1978.

2. Mercer, A. A variant of Jensen's inequality. J. Inequal. Pure Appl. Math. 2003, 4, 73.

3. Dragomir, S.S. Some reverses of the Jensen inequality for functions of self-adjoint operators in Hilbert spaces. J. Inequal. Appl. 2010, 15, 496821. [CrossRef]

4. Simić, S. On a converse of Jensen's discrete inequality. J. Inequal. Appl. 2009, 2009, 153080. [CrossRef]

5. Simic, S. Another converse of Jensen's inequality. Res. Rep. Collect. 2009, 12. [CrossRef]

6. Simić, S. On a converse of Ky Fan inequality. Krag. J. Math. 2010, 33, 95-99.

7. Stolarsky, K.B. Generalizations of the logarithmic mean. Math. Mag. 1975, 48, 87-92. [CrossRef] 